RICYDE. Revista Internacional de Ciencias del Deporte doi: $10.5232 /$ ricyde

Rev. int. cienc. deporte

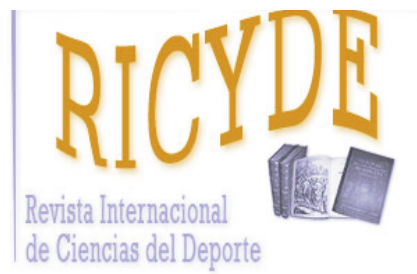

RICYDE. Revista Internacional de Ciencias del Deporte VOLUMEN XII - AÑO XII

Páginas:220-233 ISSN:1885-3137

Número 45 - Julio - 2016

\title{
La obra y el pensamiento deportivo de Manuel Vázquez Montalbán (1960-2003) The work and sports thought of Manuel Vázquez Montalbán (1960-2003)
}

\author{
Jordi Osúa, Javier Olivera \\ Instituto Nacional de Educación Física de Cataluña, Centro de Barcelona, España
}

\begin{abstract}
Resumen
La finalidad de este estudio consiste en recopilar los escritos dedicados al deporte en la prolífica obra de Manuel Vázquez Montalbán (1960-2003), valorar la existencia de una obra deportiva montalbaniana y delimitar los principales temas tratados para desentrañar las bases de su pensamiento deportivo. A pesar de la importancia de su obra en torno al análisis del deporte como un elemento de la cultura popular y como una potente herramienta para descifrar los procesos sociales, los estudios académicos dedicados a investigar esta dimensión de su obra y de su pensamiento son escasos. La compilación de sus escritos deportivos se realiza a partir del análisis sistemático de sus artículos catalogados en trabajos anteriores y de su obra literaria publicada. Las principales líneas de su pensamiento deportivo se establecen en categorías temáticas a partir del cálculo de la frecuencia de aparición en el conjunto de textos deportivos. Los resultados obtenidos confirman la existencia de una obra deportiva original formada por 700 escritos, en su mayoría artículos periodísticos. Las categorías temáticas más importantes están relacionadas con el análisis social, político, mediático y económico del deporte; la crítica marxista; la significación política y social del Barça; o el patriotismo deportivo. Esta investigación aporta el descubrimiento de 178 artículos deportivos no catalogados anteriormente y la delimitación del pensamiento deportivo montalbaniano en torno a tres aspectos: las connotaciones políticas y sociales del fútbol español, la desvirtuación política y económica del deporte y la reivindicación del simbolismo del F. C. Barcelona.
\end{abstract}

Palabras clave: Manuel Vázquez Montalbán; obra deportiva; pensamiento deportivo; política y deporte; fútbol español; F. C. Barcelona; crítica marxista.

\begin{abstract}
The aim of this study consists on compiling the texts devoted to sport in the prolific career of Manuel Vázquez Montalbán (1960-2003), to value the existence of a 'montalbanian' sport work and to delimit the main discussed themes to unravel the basis of his sports thought. Despite the importance of his works regarding the analysis of sport as a popular cultural element and as a powerful tool to decipher social processes, academic studies devoted to research on this dimension of his works and thought are scarce. The compiling of his writings on sports is carried out from the systematic analysis of his catalogued articles in previous writings and of his published literary works. His main lines of sport thought are established in thematic categories from the calculation of the frequency in publication on the whole of sport texts. The obtained results confirm the existence of an original sport work made up of 700 texts, mostly journalistic articles. The most important thematic categories are related to the social, political, media and economical analysis of sport; the Marxist criticism; the political and social significance of Barça; or the sport patriotism. This study reveals the discovery of 178 non previously catalogued sport articles and the delimitation of the 'montalbanian' sport thought around three aspects: political and social connotations of Spanish football, the political and economical distortion of sport and the recognition of FC Barcelona symbolism.
\end{abstract}

Key words: Manuel Vázquez Montalbánsport works; sport thought; politics and sport; Spanish football; F.C . Barcelona; Marxist criticism. 


\section{Introducción}

$M$ anuel Vázquez Montalbán puede ser considerado uno de los escritores españoles contemporáneos más importantes. Novelista, poeta, ensayista y periodista, su obra abarca temas como la política, la gastronomía, la literatura, la música o el deporte. Su obra es muy prolífica, casi 150 libros y más de 9000 artículos periodísticos publicados (Salgado, 2009), y variada, tanto en los temas tratados como en los géneros literarios utilizados. Por su talento le reconocieron con el premio Planeta (1979), el premio Nacional de Literatura (1991), el premio Nacional de la Crítica (1995) y el premio Nacional de Narrativa y de las Letras (1995).

La gran mayoría de los estudios sobre la obra de Manuel Vázquez Montalbán se han desarrollado desde el ámbito literario o comunicativo. Pero el reconocimiento público del que gozó Vázquez Montalbán como intelectual y escritor, no se corresponde con las investigaciones sobre su vida, su obra y su pensamiento iniciadas en España únicamente a raíz de su muerte (Rey, 2005; Salgado, 2009; Osúa, 2013). Sin embargo, en el ámbito internacional su obra y su pensamiento habían sido estudiados con anterioridad, tanto en lo que respecta a su narrativa (Colmeiro, 1996; Balibrea, 1999; ${ }^{1}$ como a investigaciones sobre algún aspecto de su escritura, como por ejemplo los pseudónimos periodísticos empleados (Erba, 1998), su poesía (Oriol-Barcelo, 2001; Estrade, 2006) o su novela policiaca (Tyras, 1992; Courthieu, 2001; Camilleri, 2004).

No hay ninguna duda a la hora de reconocer que Vázquez Montalbán fue un gran novelista y periodista, pero otras dimensiones de su reflexión intelectual, relacionadas con algunas de sus aficiones más personales, como la gastronomía o el deporte, no han sido estudiadas en profundidad. Pese a definirse a sí mismo como "periodista, novelista, poeta, ensayista, antólogo, prologuista, humorista, crítico, gastrónomo, culé y prolífico en general”, el contenido de los artículos dedicados a glosar la figura de Vázquez Montalbán con motivo de su muerte denota el olvido de la dimensión deportiva en su obra por parte de algunos especialistas. Por un lado, destaca la no inclusión de un apartado específico dedicado al deporte en la descripción de su biblioteca (Geli y Gracia, 2003; Moret, 2003). Por otro, la ausencia de referencias al proyecto de recopilación de sus escritos futbolísticos, "Fútbol. Una religión en busca de un dios", en los artículos periodísticos publicados al día siguiente de su muerte, el 19 de octubre de $2003 .^{2}$

Aun así, en las dos tesis doctorales españolas dedicadas a otros aspectos de la obra montalbaniana, el deporte aparece como uno de los aspectos específicos de la misma. Pablo Rey (2005) demuestra en su análisis temático que el fútbol constituye una categoría temática cuantitativamente significativa en su obra periodística y literaria y un tema de especial interés para Manuel Vázquez Montalbán. Francesc Salgado (2009) incluye en el catálogo periodístico elaborado entre 1960 y 1978 un total de 77 artículos, 38 englobados bajo la categoría "deportes" y 39 en "fútbol", y aglutina sus reflexiones en torno a dos ejes ideológicos: la utilización del deporte como un instrumento de alienación y la interpretación simbólica del significado del F. C. Barcelona.

\footnotetext{
${ }^{1}$ Libro dedicado a su tesis doctoral defendida en la Universidad de California, San Diego, en 1996.

${ }^{2}$ Gràcies, M.V.M. Avui, p. 2; L’infatigable Vázquez Montalbán mor a Bangkok. Avui, p. 47; Se rompe el corazón de Vázquez Montalbán. El País, p. 38.
} 
Por otro lado, Joan Rius (2004) presentó, por primera vez y con motivo de su muerte, en un artículo divulgativo las principales ideas relacionadas con el mundo del deporte expuestas por Manuel Vázquez Montalbán en su obra en torno a seis ejes temáticos: la crítica a los intelectuales, la crítica a Coubertin, el paso del juego al deporte politizado, deporte y mito, la influencia en las teorías del deporte, y el deporte y los Juegos Olímpicos. Dos años después (Rius, 2006) amplió este trabajo con un apartado dedicado a la historia del deporte y al concepto montalbaniano de educación física y deporte, otro a la definición del deporte, tanto el fútbol como el olimpismo, como religión moderna, y un tercero a la denuncia de los contravalores del deporte vinculados al capitalismo. Asimismo, Carles Geli (2009) expuso en un trabajo de máster inédito las ideas principales relacionadas con la evolución del fútbol hasta convertirse en una religión de diseño a partir de unos cuantos artículos periodísticos, algunos aspectos biográficos relacionados con este deporte y la importancia de su figura dentro del periodismo deportivo español.

Además de estos trabajos académicos, diversos autores vinculados al mundo de la Actividad Física y el Deporte han reivindicado la importancia de la obra y del pensamiento deportivo montalbaniano para el análisis del simbolismo antropológico y social del F. C. Barcelona (Burns, 1999; Colomé, 1999; Salvador, 2005; Santacana, 2005, 2008), la denuncia del rechazo del deporte por parte de los intelectuales (Alcoba, 1972; Meynaud, 1972; Cazorla, 1979; Verdú, 1980; Santacana, 2005), la consideración del deporte como un ritual religioso (Durán, 1996; Feixa, 2003), el estudio de la relación entre fútbol y política durante el franquismo (Shaw, 1987) o el origen de la agresividad en el deporte (Cagigal, 1990). Incluso, José Luis Salvador (2004) ha considerado que Manuel Vázquez Montalbán debería ser considerado por los profesionales de la Educación Física como uno de los mejores sociólogos del deporte en España.

El conjunto de estos estudios y citaciones bibliográficas, a raíz de su inesperada muerte, demuestra la importancia y el valor que en el ámbito de la Actividad Física y el Deporte pueden tener la obra y el pensamiento deportivo montalbanianos. Por este motivo, los objetivos de este estudio son tres:

1) Recopilar el conjunto de escritos dedicados al deporte en la obra de Manuel Vázquez Montalbán.

2) Cuantificar las referencias deportivas encontradas y valorar si constituyen una auténtica obra deportiva.

3) Establecer las principales líneas del pensamiento deportivo montalbaniano.

Las aportaciones más relevantes de esta investigación son la recopilación de una obra deportiva formada por 700 escritos deportivos, presentes mayoritariamente en sus artículos periodísticos pero también en los ensayos, novelas, prólogos, entrevistas y obras colectivas en las que colaboró. El descubrimiento de 178 artículos dedicados al deporte que no habían sido incluidos en ninguno de los catálogos elaborados anteriormente. La constatación de que el deporte constituye una temática habitual en la obra montalbaniana. Y la delimitación del pensamiento deportivo montalbaniano en torno a tres cuestiones: las connotaciones políticas y sociales del fútbol español, la desviación política y económica del deporte y el simbolismo del F. C. Barcelona. 


\section{Método}

El método cualitativo empleado para alcanzar los objetivos de este estudio es el historiográfico. Por tanto, en un primer momento la mayor parte del trabajo ha consistido en la recopilación y lectura sistemática de las fuentes montalbanianas (método heurístico), fundamentalmente las relacionadas con el deporte. Posteriormente, a partir de la información obtenida, se ha estructurado el pensamiento deportivo montalbaniano analizando el contenido de sus escritos y organizando, de manera interpretativa, las ideas expuestas en torno a los temas tratados (método hermenéutico). La elección de esta metodología está en la línea de la utilizada en otros estudios similares dedicados al estudio de la obra montalbaniana (Salgado, 2009) o a la de otros autores como José María Cagigal (Olivera, 2006).

La recopilación de la obra deportiva de Manuel Vázquez Montalbán se ha realizado a través de la búsqueda sistematizada de las fuentes primarias. Ante la imposibilidad de acceder a sus materiales personales para encontrar obras inéditas, la búsqueda de los escritos deportivos se circunscribirá a su obra publicada en castellano y en catalán. Esta obra incluye cinco tipos de publicaciones: a) artículos periodísticos, tanto en diarios como en revistas diversas; b) libros, ya sean ensayos, novelas, recopilaciones de artículos periodísticos, poemas u otros (guiones televisivos, obras de teatro...); c) capítulos de libros colectivos; d) prólogos de libros; y e) entrevistas, recogidas en medios audiovisuales, libros o páginas web.

La localización de los artículos deportivos publicados en diarios y revistas se ha realizado a partir de los catálogos periodísticos o compilaciones de artículos realizados hasta el momento. Primero, el catálogo elaborado por un grupo de estudiantes de Periodismo de la Universidad Pompeu Fabra, en el marco de la asignatura de "Historia del Periodismo", durante el curso 2003-2004 que contiene más de 8000 artículos escritos en 37 publicaciones diferentes entre 1960 y 2003. ${ }^{3}$ Segundo, los catálogos incluidos en las tesis doctorales de Francesc Salgado (2009), artículos publicados hasta 1978, y de Joaquim Roglán (2010), artículos publicados en la revista Bocaccio. Tercero, la selección de artículos entre 1960 y 2003 realizada en los tres volúmenes editados por Francesc Salgado (2010, 2011 y 2012) y en el libro de Carles Geli y Francesc Mauri (2008). Cuarto, los artículos publicados en la prensa internacional conservados en el archivo de la Agencia Literaria Carmen Balcells.

La búsqueda de los libros y de los capítulos de libros dedicados al deporte se ha desarrollado a través de las bases de datos de las bibliotecas de las universidades catalanas y del resto de España, en el Archivo ISBN del Ministerio de Cultura o en los listados exhaustivos incluidos en las biografías elaboradas hasta el momento (Saval, 2004; Estrade, 2004), en el trabajo de Carles Geli (2009) y en la tesis doctoral de Pablo Rey (2005). En cuanto a los prólogos no existe ningún trabajo de catalogación previo, aunque Florence Estrade (2004) dedica en su libro un apartado a este género literario y en la bibliografía incluye algunos de ellos, y en "vespito", la página web "oficiosa" dedicada al escritor barcelonés, aparecen muchos de sus prólogos. Por último, las entrevistas que concedió a diversos medios de comunicación audiovisuales, radio y televisión, y escritos, diarios y revistas, se encuentran en las bases de datos de las bibliotecas universitarias, en las publicaciones periódicas donde colaboró y en la página web "vespito".

\footnotetext{
3 Alumnos asignatura Historia del Periodismo de la Universidad Pompeu Fabra (curso 2003-2004). Catálogo obra periodística de Manuel Vázquez Montalbán. Tomado en febrero, 25, 2011.

http://www.upf.edu/depeca/depeca/mvm/index3.htm.

${ }^{4}$ Tomado en mayo, 4, 2011. http://www.vespito.net.
} 
Una vez determinadas las fuentes montalbanianas y la manera de acceder a ellas se han seleccionado todos los escritos que formarán parte de la obra deportiva. Esta elección se ha llevado a cabo teniendo en cuenta una serie de criterios aplicados de manera excluyente en función del siguiente orden: 1) el título deportivo, 2) la sección o el libro deportivo, 3) el contenido deportivo, 4) los apuntes o comentarios deportivos, y 5) los textos deportivos incluidos en otras publicaciones periódicas no catalogadas.

Finalmente, para esclarecer las principales líneas del pensamiento deportivo montalbaniano se ha procedido al análisis del contenido y a la elaboración de una ficha hermenéutica de cada uno de los escritos deportivos. Dentro de los diferentes tipos de análisis de contenido se ha escogido el análisis temático categorial consistente en calcular y comparar la frecuencia de aparición de las categorías temáticas definidas (Quivy y Campenhoudt, 2004). Este análisis ha permitido establecer los temas deportivos tratados preferentemente por Vázquez Montalbán. En la ficha hermenéutica se han anotado las ideas más importantes y las citas más significativas con relación a los objetivos propuestos (Olivera, 2006).

\section{Resultados}

Tras revisar pormenorizadamente el conjunto de la obra publicada de Manuel Vázquez Montalbán y aplicar los criterios de selección expuestos con anterioridad se han encontrado un total de 700 escritos dedicados al deporte. Las referencias localizadas en función del título deportivo son 389 (55.5\%), de la sección periodística o del libro deportivo son 40 (5.7\%), del contenido deportivo son 155 (22.2\%), de los apuntes o comentarios deportivos son 66 (9.5\%) y de otras publicaciones distintas a las catalogadas son 50 (7.1\%).

En cuanto al tipo de publicación, en la Tabla 1 se exponen la cantidad de referencias deportivas correspondientes a artículos periodísticos, libros, capítulos de libros, prólogos y entrevistas teniendo en cuenta la temática deportiva o no de dichas publicaciones. El 89.7\% de los textos deportivos (628) tienen un contenido o un título explícitamente deportivo, mientras que solamente el $10.3 \%$ (72) corresponden a capítulos de libro o comentarios aparecidos en publicaciones dedicadas a otra temática diferente.

Tabla 1. Referencias deportivas encontradas en función del tipo de publicación.

\begin{tabular}{|l|c|c|}
\hline Tipo de publicación & \multicolumn{2}{|c|}{ Referencias deportivas } \\
\hline \multirow{2}{*}{ Artículos periodísticos de temática deportiva } & \multicolumn{2}{|c|}{2} \\
\hline \multirow{2}{*}{ Libros de temática deportiva } & Ensayos & \multicolumn{2}{|c|}{2} \\
\cline { 2 - 3 } & Novelas & 2 \\
\cline { 2 - 3 } & Guiones & 5 \\
\hline Prólogos de temática deportiva & Poemas & 5 \\
\hline Capítulos de libro de temática deportiva & 4 \\
\hline Entrevistas de temática deportiva & 6 \\
\hline Capítulos deportivos en libros no deportivos & 14 \\
\hline Apuntes deportivos en libros no deportivos & 45 \\
\hline Apuntes deportivos en artículos no deportivos & \\
\hline
\end{tabular}


Osúa, J., y Olivera, J. (2016). La obra y el pensamiento deportivo de Manuel Vázquez Montalbán (1960-2003). RICYDE. Revista internacional de ciencias del deporte. 45(12), 220-233.

\begin{tabular}{|l|c|}
\hline Apuntes deportivos en entrevistas no deportivas & 7 \\
\hline Total referencias deportivas & 700 \\
\hline
\end{tabular}

El 93\% de estos escritos deportivos (648) son artículos publicados en diferentes diarios y revistas. En total se han encontrado referencias deportivas en 48 publicaciones periódicas diferentes, aunque en 27 de ellas solo escribió un texto deportivo. El 71.1\% (461) de estos artículos pertenecen a cuatro publicaciones: El País (225), El Periódico de Cataluña (98), Interviú (74) y Triunfo (64). Además, si ampliamos esta selección a Avui (47), Por Favor (43), Tele/Exprés (12), La Calle (11), La Reppublica (9), Barça (8) e Il Manifesto (7) vemos como el 92.2\% (598) de los artículos se concentran en 11 publicaciones.

El primer escrito deportivo localizado corresponde a un artículo publicado en El Español el 18 de septiembre de 1960 y el último al libro póstumo "Fútbol. Una religión en busca de un Dios" publicado en 2005. Entre 1969, ${ }^{5}$ año de su eclosión periodística, y 2003, cuando falleció, Vázquez Montalbán abordó cuestiones deportivas con una gran regularidad. La Tabla 2 muestra como durante estos 35 años solo en tres de ellos (1969, 1986 y 1987) publicó menos de diez textos deportivos. En cambio aparecen cinco años con más de treinta escritos deportivos (1981, 1992, 1997,1998 y 2002) y otros cinco años con más de veinticinco (1977, 1980, 1982, 1988 y 1992).

Respecto a su distribución por décadas no existen grandes diferencias y la media anual tanto en los años setenta como en los ochenta y los noventa oscila entre 17 y 23 escritos deportivos, aumentando progresivamente. Entre los años 2000 y 2003 la producción deportiva continúa siendo de más de veinte textos por año.

Tabla 2. Distribución de las referencias deportivas por año.

\begin{tabular}{|c|c|c|c|c|c|}
\hline Año & Referencias & Año & Referencias & Año & Referencias \\
\hline 1969 & 5 & 1981 & 32 & 1993 & 13 \\
\hline 1970 & 14 & 1982 & 29 & 1994 & 22 \\
\hline 1971 & 15 & 1983 & 16 & 1995 & 16 \\
\hline 1972 & 23 & 1984 & 18 & 1996 & 28 \\
\hline 1973 & 14 & 1985 & 18 & 1997 & 31 \\
\hline 1974 & 16 & 1986 & 7 & 1998 & 37 \\
\hline 1975 & 12 & 1987 & 6 & 1999 & 19 \\
\hline 1976 & 10 & 1988 & 25 & 2000 & 22 \\
\hline 1977 & 27 & 1989 & 21 & 2001 & 12 \\
\hline 1978 & 22 & 1990 & 14 & 2002 & 32 \\
\hline 1979 & 20 & 1991 & 14 & 2003 & 15 \\
\hline 1980 & 27 & 1992 & 41 & & \\
\hline
\end{tabular}

\footnotetext{
5 Entre 1960 y 1969 únicamente aparecen cinco referencias deportivas como consecuencia de su detención y encarcelamiento en 1962 y la precariedad laboral que vivió, con la retirada del carné de periodista y los antecedentes penales, tras salir de la cárcel en 1963.
} 
El análisis de contenido muestra la existencia de 16 categorías temáticas con un frecuencia de aparición superior al $14.2 \%$, es decir, presentes en más de 100 escritos deportivos, una cifra bastante significativa. En la Tabla 3 aparecen cada uno de estos temas ordenados en función de su importancia en el conjunto de la obra deportiva. Por encima del resto destacan tres categorías: "política nacional y deporte" (43.4\%), "memoria deportiva" (38.4\%) y "medios de comunicación" (36.4\%). Además, también son significativas las categorías temáticas relacionadas con los aspectos económicos, "economía y deporte" (25.9\%); la crítica marxista, "héroe deportivo" (25.6\%), "compensación deportiva" (24.1\%), "mistificación deportiva" (15.6\%) y "alienación (público)"; el simbolismo del F. C. Barcelona (22.4\%); la política internacional (18.6\%) y la rivalidad entre el F. C. Barcelona y el Real Madrid (15.7\%).

Tabla 3. Categorías temáticas con una frecuencia de aparición superior al $14.2 \%$ en el conjunto de la obra deportiva.

\begin{tabular}{|l|c|}
\hline Categoría temática & Frecuencia de aparición \\
\hline Política nacional y deporte & $43.4 \%$ \\
\hline Memoria deportiva & $38.4 \%$ \\
\hline Medios de comunicación & $36.4 \%$ \\
\hline Política de club & $27.1 \%$ \\
\hline Economía y deporte & $25.9 \%$ \\
\hline Héroe deportivo & $25.6 \%$ \\
\hline Compensación deportiva & $24.1 \%$ \\
\hline Análisis futbolístico & $22.9 \%$ \\
\hline Público en general & $22.7 \%$ \\
\hline Significación política y social del F. C. Barcelona & $22.4 \%$ \\
\hline Patriotismo deportivo español & $19.1 \%$ \\
\hline Política internacional y deporte & $18.6 \%$ \\
\hline Antagonismo deportivo F. C. Barcelona-Real Madrid & $15.7 \%$ \\
\hline Mistificación deportiva & $15.6 \%$ \\
\hline Alienación deportiva (público) & $14.9 \%$ \\
\hline
\end{tabular}

\section{Discusión}

Durante el proceso de búsqueda y recopilación de los escritos deportivos se han encontrado numerosos artículos no incluidos en el catálogo de la Universidad Pompeu Fabra (2004) ni en el de la tesis doctoral de Francesc Salgado (2009). En el primer caso, un total de 123 artículos, debido a un error en algunas publicaciones periódicas donde se habían descuidado la catalogación de algunos años. En el segundo, un total de 8 artículos, como consecuencia de una diferencia de criterio a la hora de considerar un artículo como deportivo o no, pero también por el descuido en la catalogación de algunos de ellos.

Por otro lado, el tercer volumen de la obra periodística montalbaniana editado por Francesc Salgado (2012) puso al descubierto la existencia de artículos publicados en la prensa extranjera. A través de la Agencia Literaria Carmen Balcells, y de su secretaria Carina Pons, 
Osúa, J., y Olivera, J. (2016). La obra y el pensamiento deportivo de Manuel Vázquez Montalbán (1960-2003). RICYDE. Revista internacional de ciencias del deporte. 45(12), 220-233.

se realizaba todo el proceso de solicitud y remisión de estos artículos. Por esta razón, en los archivos de esta empresa se conservan los textos que Vázquez Montalbán escribía para ser enviados posteriormente a cada una de las publicaciones y las fichas con el registro de la fecha de salida y del cobro o no del artículo. Entre estos documentos se hallaron 37 referencias deportivas, pertenecientes en su práctica totalidad a publicaciones extranjeras, no catalogadas anteriormente (Tabla 4).

Estos escritos pertenecen mayoritariamente (34) a diarios o revistas extranjeras, ${ }^{6}$ algunos de los cuáles reproducen prácticamente el mismo texto o aprovechan artículos publicados previamente en España. En estas publicaciones internacionales dio a conocer el significado político y social del F. C. Barcelona, denunció las transformaciones urbanísticas de la ciudad condal en función de los intereses empresariales con motivo de los Juegos Olímpicos, analizó diversas cuestiones políticas, sociales y deportivas relacionadas con los Mundiales de Francia (1998) y Estados Unidos (1994) y valoró la trayectoria futbolística de Diego Armando Maradona.

Tabla 4. Artículos localizados en el archivo de la Agencia Literaria Carmen Balcells.

\begin{tabular}{|c|c|c|}
\hline Título & Publicación & Fecha \\
\hline El Barcelona F. C.: algo más que un club & Sport International & $01 / 09 / 1987$ \\
\hline Si Barcelona no existiera & CLUP & $20 / 09 / 1989$ \\
\hline Barcelona: la ciudad de los prodigios irremediables & Tages-Anzeiger & $23 / 04 / 1990$ \\
\hline Crónica sentimental de la musculatura & $\begin{array}{l}\text { Olimpiada Cultural } \\
\text { Mundo Vergag }\end{array}$ & $16 / 11 / 1990$ \\
\hline $\begin{array}{l}\text { Barcelona: Norte y Sur. Las olimpiadas han impuesto un modelo de } \\
\text { crecimiento }\end{array}$ & Süddeutsche Zeitung & $04 / 12 / 1990$ \\
\hline La ciudad inevitada & Marie France & $05 / 04 / 1991$ \\
\hline El Barcelona F.C.: algo más que un club & Merian & $14 / 10 / 1991$ \\
\hline Barcelona: de 1992 al infinito & Ronda Iberia & $20 / 05 / 1992$ \\
\hline El loro y los Juegos Olímpicos & Profil & $14 / 09 / 1992$ \\
\hline Fútbol USA: el mundial atípico & Ronda Iberia & $01 / 05 / 1994$ \\
\hline Milan 4 - Barcelona 0 ¿Qué será de nosotros, sin bárbaros? & Il Manifesto & $01 / 05 / 1994$ \\
\hline Maradona: la mano de Dios & Das Magazine & $16 / 05 / 1994$ \\
\hline Campeonato 1994: un deporte en promoción & $\begin{array}{l}\text { Internacional Press } \\
\text { Service }\end{array}$ & $01 / 07 / 1994$ \\
\hline Fútbol y desorden internacional & Il Manifesto & $01 / 07 / 1994$ \\
\hline Los futbolistas son sacrificados al amanecer & Il Manifesto & $01 / 07 / 1994$ \\
\hline Baggio 2 - España 1 & Il Manifesto & $10 / 07 / 1994$ \\
\hline
\end{tabular}

\footnotetext{
${ }^{6}$ Uno de ellos publicado previamente en una revista española.
} 
Osúa, J., y Olivera, J. (2016). La obra y el pensamiento deportivo de Manuel Vázquez Montalbán (1960-2003). RICYDE. Revista internacional de ciencias del deporte. 45(12), 220-233.

http://dx.doi.org/10.5232/ricyde2016.04501

\begin{tabular}{|c|c|c|}
\hline La liga de la legión extranjera & Estrella & 28/08/1996 \\
\hline El fútbol entre la globalización y el nacionalismo & Clarín & 24/01/1997 \\
\hline Barcelona FC- Fiorentina, ser o no ser & La Nazione & $10 / 04 / 1997$ \\
\hline Y la FIFA creó a Ronaldo & La Reppublica & $25 / 04 / 1997$ \\
\hline Ronaldo: el dios de la ingeniería genética & Max & $17 / 06 / 1997$ \\
\hline Lady Di ha muerto ¡Vivan los Juegos Olímpicos! & Il Manifesto & 04/09/1997 \\
\hline Elogio desmesurado de los vándalos & Exceso & $21 / 10 / 1997$ \\
\hline El corazón de los árbitros de fútbol & La Reppublica & $04 / 05 / 1998$ \\
\hline Real Madrid, 32 años de nostalgia & La Reppublica & $21 / 05 / 1998$ \\
\hline 1998: un campeonato para el fútbol de diseño & Desconocida & 05/06/1998 \\
\hline Campeonato Mundial de Francia: multirracialismo o muerte & La Reppublica & $10 / 07 / 1998$ \\
\hline El fútbol, Nietzsche y Le Pen & L'Europeen & $10 / 07 / 1998$ \\
\hline Francia 1998: la victoria del mestizaje & Reporter & $15 / 07 / 1998$ \\
\hline $\begin{array}{l}\text { Un drama de la globalización. Los problemas de identidad del Club } \\
\text { de Fútbol Barcelona }\end{array}$ & La Reppublica & $05 / 11 / 1999$ \\
\hline Ronaldo o la rodilla de Dios & La Reppublica & $17 / 04 / 2000$ \\
\hline Los Juegos Olímpicos de la globalización & La Reppublica & $24 / 08 / 2000$ \\
\hline Figo. Traidor, inconfeso y mártir & Max & $13 / 06 / 2001$ \\
\hline Barcelona: fútbol, política y caos & La Reppublica & $13 / 03 / 2002$ \\
\hline Ronaldo y Cúper, ni perdedores ni ganadores, sino todo lo contrario & La Reppublica & $09 / 05 / 2002$ \\
\hline Mundiales 2002: a por la globalización & Il Manifesto & $07 / 06 / 2002$ \\
\hline Ronaldo: el retorno del dios remendado & Il Manifesto & $18 / 12 / 2002$ \\
\hline
\end{tabular}

Diversos autores coinciden en la imposibilidad de recopilar todos los artículos publicados de Vázquez Montalbán (Geli y Mauri, 2008; Salgado, 2009), ya que nunca negó un artículo a quien se lo pidiese, pudiéndose hallar escritos suyos en revistas de diferentes asociaciones. De esta forma, se han encontrado un total de 178 artículos no incluidos en ninguno de los dos catálogos periodísticos elaborados hasta el momento, destacando los 93 correspondientes a El Periódico de Cataluña y los 37 localizados en la Agencia Literaria Carmen Balcells (Tabla 5). 
Osúa, J., y Olivera, J. (2016). La obra y el pensamiento deportivo de Manuel Vázquez Montalbán (1960-2003). RICYDE. Revista internacional de ciencias del deporte. 45(12), 220-233. http://dx.doi.org/10.5232/ricyde2016.04501

Tabla 5. Publicaciones periódicas donde se han encontrado artículos no catalogados con anterioridad.

\begin{tabular}{|l|c|}
\hline Publicación periódica & Artículos no catalogados \\
\hline El Periódico de Cataluña & 93 \\
\hline Interviú & 16 \\
\hline Triunfo & 8 \\
\hline El País & 8 \\
\hline Barça & 3 \\
\hline Por favor & 1 \\
\hline Avui & 1 \\
\hline Bocaccio & 1 \\
\hline Mundo obrero & 1 \\
\hline Oriflama & 178 \\
\hline Cuadernos para el diálogo & 37 \\
\hline Agencia literaria Carmen Balcells & 1 \\
\hline Total artículos no catalogados & \\
\hline
\end{tabular}

La concentración de los artículos deportivos en unas publicaciones determinadas no responde a una intencionalidad concreta ni a la temática deportiva del medio, pues exceptuando la revista Barça, el resto de publicaciones son generalistas. Vázquez Montalbán no escogió un medio de comunicación específoco para compartir sus reflexiones deportivas sino que las introdujo allí donde colaboraba, independientemente de su inclusión en secciones de opinión, cultura, televisión, política nacional o política internacional. Este hecho refleja una concepción del fenómeno deportivo como un medio para analizar la sociedad del momento. Por eso, en casi todos los diarios o revistas donde colaboraba regularmente se ha encontrado algún artículo deportivo. Solamente en dos publicaciones escribió en un espacio específicamente dedicado al deporte: en Triunfo, utilizando el pseudónimo Luis Dávila, y en El País, colaborando con la sección de deportes.

Además de artículos periodísticos, la obra deportiva de Vázquez Montalbán contiene ensayos, recopilaciones de artículos, poemas, cuentos o guiones cinematográficos. En los estudios dedicados a su biografía o a su obra literaria que contienen una bibliografía extensa (Erba, 1998; Saval, 2004) no aparecen algunas de estas publicaciones deportivas como el ensayo "Calcio, una religione alla ricerca dil suo Dio"; los guiones televisivos y de películas "Delantero" y "El fantasma del estadio"; o los poemas "Bíceps, tríceps..." y "La modernidad adosó un squash".

La lectura sistemática de su obra literaria ha permitido el descubrimiento de hasta 6 capítulos deportivos en ensayos o relatos literarios dedicados a otras temáticas. Además, una parte de los textos deportivos montalbanianos aparecen en libros donde reflexiona sobre otros aspectos comunicativos, políticos, ideológicos, históricos o culturales. Hasta en 14 ensayos, novelas, recopilaciones de artículos, biografías, diccionarios o cancioneros, Vázquez Montalbán analiza cuestiones relacionadas con el deporte. Esta capacidad para relacionar elementos de la 
realidad social aparentemente inconexos constituye una característica de su estilo periodístico y literario presente, sobre todo, en sus libros.

También hallamos referencias al deporte en las colaboraciones que Vázquez Montalbán mantuvo con otros autores (prólogos y obras colectivas), en las entrevistas o en las biografías elaboradas hasta el momento. Las reflexiones sobre distintos aspectos de la realidad deportiva contenidas en sus escritos deportivos despertaron el interés, la admiración y el respeto de algunos autores que solicitaron la escritura de un prólogo o su participación en un libro colectivo. Los prólogos "Vándalos y vándalos" y "El Barça: de la guerra civil a la casa real" no se incluyen en los listados bibliográficos existentes. Por otro lado, el deporte constituía una de sus aficiones más conocidas y uno de los ámbitos culturales presentes en sus actividades profesionales. Por eso, se convirtió en un aspecto singular incluido en las entrevistas concedidas a diversos medios audiovisuales y escritos o en los libros biográficos.

El análisis de contenido ratifica las conclusiones de los estudios realizados sobre la importancia de algunos de los temas tratados por Vázquez Montalbán en su obra deportiva. Por ejemplo, las connotaciones políticas del deporte en España durante el franquismo, especialmente del fútbol de clubes y de la selección española, para fomentar el patriotismo español y desviar la atención de los problemas sociales (Salgado, 2009; Osúa, 2013). La memoria histórica (Geli, 2009); la pérdida de los valores deportivos debido a su comercialización (Rius, 2006); la denuncia de la alienación del público, del engaño oculto en la ideología deportiva y de la politización de las grandes competiciones internacionales (Salgado, 2009; Osúa, 2013); los comentarios futbolísticos (Geli, 2009); o la interpretación simbólica del F. C. Barcelona (Salgado, 2009; Osúa, 2013) corresponden a otras temáticas que abordó con frecuencia.

En cambio, la mayoría de los temas expuestos por Joan Rius $(2004,2006)$ son relevantes en algún momento de la trayectoria intelectual de Manuel Vázquez Montalbán, pero no resultan tan significativos en el conjunto de su obra deportiva, seguramente porque responden al contenido de dos de sus libros de ensayo publicados a principios de los años setenta.

Pero mediante el análisis de contenido destacamos otros temas no especificados en los anteriores trabajos como la utilización política del fútbol y del deporte español por parte de los gobiernos democráticos (ucedistas, socialistas y populares) y de los medios de comunicación españoles, sobre todo Radio Televisión Española pero también los medios privados, durante el franquismo y la Transición. La transformación del deporte, del fútbol y del olimpismo como consecuencia de su comercialización y de la entrada de la publicidad; la participación pasiva del público en la gesta deportiva como una alternativa para compensar la imposibilidad de triunfar en la vida; o la interpretación de la rivalidad entre el F. C. Barcelona y el Real Madrid como un reflejo de las tensiones políticas y sociales en España son algunas de las temáticas reflejadas en sus trabajos.

Las reflexiones y comentarios recogidos en las fichas hermenéuticas elaboradas para cada uno de los escritos deportivos reflejan un pensamiento deportivo montalbaniano basado en cinco aspectos. Primero, la crítica marxista para denunciar la desviación política y económica del deporte al servicio de los gobiernos y de las empresas hasta convertirse en un negocio, aprovechándose de la participación religiosa del público con la victoria a través del deportista. Segundo, el análisis subcultural para defender el valor individual (compensación épica) y social (participación social, identidad y expresión de la sentimentalidad popular) que los espectáculos deportivos tienen para el público. Tercero, el significado político y social del Barça como instrumento de reivindicación de una identidad nacional y de cohesión e 
integración social. Cuarto, el valor del pasado deportivo para mantener viva la memoria histórica del franquismo, recuperar las vivencias colectivas y evidenciar las contradicciones entre la ideología y la realidad deportiva. Quinto, el fútbol español se convierte en el principal escenario donde estudiar las transformaciones políticas, económicas, sociales e ideológicas de la sociedad española des del franquismo hasta la modernización del país.

\section{Conclusiones}

La recopilación de la obra deportiva montalbaniana evidencia la existencia de un corpus de escritos relacionados con el deporte suficientemente importante como para ser tenido en cuenta en los estudios pertenecientes a las Ciencias de la Actividad Física y el Deporte. Vázquez Montalbán incluye su reflexión deportiva en todos los géneros literarios y periodísticos que cultivó durante su extensa y prolífica trayectoria como escritor y articulista. Las 700 referencias deportivas encontradas le convierten en uno de los escritores e intelectuales españoles que más atención ha dispensado a este fenómeno social propio de las sociedades modernas.

Su crítica marxista del deporte no responde a una perspectiva ideologizada sino a un interés vital gestando un pensamiento propio alejado del discurso ortodoxo y partidista; defiende el simbolismo político y social del Barça adquirido durante el franquismo, mostrando una nostalgia por un barcelonismo sentimental; y explica el papel del fútbol de clubes en la sociedad española para canalizar conflictos políticos y sociales durante el franquismo y garantizar la convivencia en el Estado español democrático. Por tanto, el pensamiento deportivo de Vázquez Montalbán se centra en el análisis de la relación entre deporte y sociedad, entendiendo el deporte como un producto de la realidad política, económica y social $\mathrm{y}$, a su vez, como una herramienta para descifrar los procesos sociales.

A tenor del número significativo de referencias deportivas recopiladas, de la diversidad y profundidad de los temas tratados y de la perspectiva de análisis utilizada podemos afirmar que, si bien el deporte no puede considerarse uno de los principales objetivos de su obra, su aportación en este ámbito de conocimiento resulta significativa y continuada en el tiempo. Así pues, la obra y el pensamiento deportivo montalbanianos deberían ser un referente a la hora de abordar algunas cuestiones teóricas, históricas, antropológicas y sociológicas en el campo de la Actividad Física y el Deporte.

\section{Referencias}

Alcoba, A. (1972). ¿Contamos contigo?. Madrid: G. del Toro.

Balibrea, M. P. (1999). En la tierra baldía: Manuel Vázquez Montalbán y la izquierda española en la posmodernidad. Barcelona: El Viejo Topo.

Burns, J. (1999). Barça, la pasión de un pueblo. Barcelona: Anagrama/Empúries.

Cagigal, J. (1990). Deporte y agresión. Madrid: Alianza Deporte.

Camilleri, A. (2004). Quando il Mediterraneo si tinge di giallo: Manuel Vázquez Montalbán (Tesis doctoral inédita). Università degli Studi di Bergamo, Bergamo.

Cazorla, L. (1979). Deporte y Estado. Barcelona: Labor.

Colmeiro, J. (1996). Crónica del desencanto: la narrativa de Manuel Vázquez Montalbán. Florida: Nort South Center Press, University of Miami.

Courthieu, C. (2001). Les fictions policières de Manuel Vázquez Montalbán: vingt-cinq ans de chronique sociale et de création littéraire, 1972-1997 (Tesis doctoral inédita). Université de Toulouse-Le Mirail, Toulouse. 
Durán, J. (1996). Espectáculos deportivos, sociedades democráticas y economía de mercado. En R. Sánchez (Ed.), La actividad física y el deporte en un contexto democrático (19761996) (pp. 27-34). Pamplona. AEISAD.

Erba, R. (1998). Pseudónimos y censura en la obra periodística de Manuel Vázquez Montalbán (Tesis doctoral inédita). Università di Milano, Milán.

Estrade, F. (2004). Manuel Vázquez Montalbán. Barcelona: La Tempestad.

Estrade, F (2006). L'oeuvre poétique de Manuel Vázquez Montalbán (1939-2003) (Tesis doctoral inédita). Université Paris-Sorbonne, París.

Feixa, C. (2003). Un antropólogo en el fútbol. En F. X. Medina y R. Sánchez (Eds.), Culturas en juego. Ensayos de antropología del deporte en España (pp. 73-102). Barcelona: Icaria.

Geli, C. (2009). Futbol: d'opi del poble a perfum intel-lectual (Proyecto de máster inédito). Universitat Ramon Llull, Barcelona.

Geli, C., y Gracia, J. (19 de octubre de 2003). Una biblioteca brillante, plural y extensa. El Periódico, p. 9.

Geli, C., y Mauri, M. (2008). El periodismo según Manuel Vázquez Montalbán. Barcelona: Ronsel.

Meynaud, J. (1972). El deporte y la política. Análisis social de unas relacionas ocultas. Barcelona: Hispano Europea.

Moret, X. (19 de octubre de 2003). Carvalho, Galíndez y mucho más. El País, p. 39.

Olivera, J. (1996). José María Cagigal Gutiérrez (1928-1983). Vida, obra y pensamiento en torno a la Educación Física y el Deporte (Tesis doctoral inédita). Universidad de Barcelona-INEFC, Barcelona.

Oriol-Barcelo, A. (1998). De l'espace barcelonais à l'espace textuel: l'itinéraire d'un poête-romancier, Manuel Vazquez Montalban (Tesis doctoral inédita). Université Paris-Sorbonne, París.

Osúa, J. (2013). El deporte en la vida y en la obra de Manuel Vázquez Montalbán (19392003) (Tesis doctoral inédita). Universidad de Barcelona-INEFC, Barcelona.

Rey, P. (2005). Análisis temático de la obra narrativa y periodística de Manuel Vázquez Montalbán (Tesis doctoral inédita). Universidad Pontificia de Salamanca, Salamanca.

Rius, J. (2004). El deporte para M. Vázquez Montalbán. Apunts. Educación Física y Deportes (75), 92-97.

Rius, J. (2006). Paseos por el deporte (en memoria de Manuel Vázquez Montalbán). Barcelona: Col·legi de Llicenciats en Educació Física i Ciències de I'Activitat Física i l'Esport de Catalunya.

Roglán, J. (2010). La revista Bocaccio i la construcció periodística de la Gauche Divine (Tesis doctoral inédita). Universitat Ramon Llull, Barcelona.

Salgado, F. (2009). La construcció de la identitat periodística de Manuel Vázquez Montalbán. De la censura a la transició (1960-1978) (Tesis doctoral inédita).. Universitat Pompeu Fabra, Barcelona.

Salgado, F. (2010). Manuel Vázquez Montalbán. Obra periodística I (1960-1973). La construcción del periodista. Barcelona: Debate.

Salgado, F. (2011). Manuel Vázquez Montalbán: obra periodística II (1974-1986). Del humor al desencanto. Barcelona: Debate. 
Salgado, F. (2012). Manuel Vázquez Montalbán: obra periodística III (1987-2003). Las batallas perdidas. Barcelona: Debate.

Salvador, J. (2005). Fútbol: metàfora d'una guerra freda. Barcelona: Proa.

Salvador, J. L. (2004). El deporte en Occidente. Historia, cultura y política. Madrid: Cátedra.

Santacana, C. (2005). El Barça i el franquisme. Barcelona: Mina.

Santacana, C. (2008). Pensant l'esport: els intel-lectuals i l'esport a Catalunya. En C. Santacana (Coord.), L'esport a Catalunya. Nadala 2008 (pp. 30-43). Barcelona: Fundació Lluís Carulla.

Saval, J. V. (2004). Manuel Vázquez Montalbán. El triunfo de un luchador incansable. Madrid: Síntesis.

Shaw, D. (1987). Fútbol y franquismo. Madrid: Alianza.

Tyras, G. (1992). Des traces de Pepe Carvalho (Tesis doctoral inédita). Université Stendhal, Grenoble.

Verdú, V. (1980). El fútbol. Mitos, ritos y símbolos. Madrid: Alianza. 